\section{Probing pancreatic disease using tissue optical spectroscopy}

\author{
Malavika Chandra, a James Scheiman,, ${ }^{b, c}$ David Heidt, \\ Diane Simeone, ${ }^{c, d}$ Barbara McKenna, ${ }^{e}$ and \\ Mary-Ann Mycek ${ }^{\mathrm{a}, \mathrm{c}, \mathrm{f}}$, \\ ${ }^{a}$ University of Michigan, Applied Physics Program \\ ${ }^{b}$ University of Michigan, Department of Internal Medicine \\ 'University of Michigan, Comprehensive Cancer Ctr. \\ dUniversity of Michigan, Department of Surgery \\ eUniversity of Michigan, Department of Pathology \\ fUniversity of Michigan, Department of Biomedical \\ Engineering, Ann Arbor, Michigan 48109
}

Abstract. Pancreatic adenocarcinoma, one of the leading causes of cancer death in the United States, has a five-year survival rate of only $4 \%$. Present detection methods do not provide accurate diagnosis in the disease's early stages. To investigate whether optical spectroscopy could potentially aid in early diagnosis and improve survival rates, reflectance and fluorescence spectroscopies were employed for the first time in a limited pilot study to probe freshly excised human pancreatic tissues (normal, pancreatitis, and adenocarcinoma) and in vivo human pancreatic cancer xenografts in nude mice. In human pancreatic tissues, measurements were associated with endogenous fluorophores $\mathrm{NAD}(\mathrm{P}) \mathrm{H}$ and collagen, as well as tissue optical properties, with larger relative collagen content detected in adenocarcinoma and pancreatitis than normal. Good correspondence was observed between spectra from adenocarcinoma and cancer xenograft tissues. Reflectance data indicated that adenocarcinoma had higher reflectance in the 430- to 500-nm range compared to normal and pancreatitis tissues. The observed significant differences between the fluorescence and reflectance properties of normal, pancreatitis, and adenocarcinoma tissues present an opportunity for future statistical validation on a larger patient pool and indicate a potential application of multimodal optical spectroscopy to differentiate between diseased and normal pancreatic tissue states. ๑ 2007 Society of Photo-Optical Instrumentation Engineers. [DOI: 10.1117/1.2818029]

Keywords: fluorescence; reflectance; tissue spectroscopy; carcinoma; pancreas; time resolved.

Paper 07258LR received Jul. 19, 2007; revised manuscript received Aug. 30, 2007; accepted for publication Sep. 3, 2007; published online Dec. 14, 2007.

Pancreatic adenocarcinoma is the fourth leading cause of cancer death in the United States. ${ }^{1}$ This dismal prognosis results from most symptomatic patients having unresectable disease at clinical evaluation, leading to a five-year survival rate of $4 \%{ }^{1,2}$ Current diagnostic methods, including computed tomography $(\mathrm{CT})$, magnetic resonance imaging (MRI), and endoscopic ultrasound (EUS), have not been able to provide accurate diagnosis in early stage disease, ${ }^{1}$ either by failing to identify small lesions or accurately differentiating masses as

Tel.: 734-647-1361; E-mail: mycek@umich.edu either adenocarcinoma or pancreatitis (inflammation of the pancreas). These challenges occur due to the relative inaccessibility of the pancreas given its anatomical location, the nonspecific nature of symptoms (pain and weight loss), as well as the characteristic stromal reaction with intense fibrosis associated with both adenocarcinoma and pancreatitis. ${ }^{3}$ This significantly complicates attempts to differentiate the two similar appearing lesions by imaging, even with cytological evaluation of needle aspirates. ${ }^{4}$ Consequently, patients may undergo major surgery to reveal only benign or inflammatory disease on pathologic examination. One study reported that as many as $9.2 \%$ of surgeries were negative for neoplastic disease, even though they had been performed for a clinical suspicion of malignancy. ${ }^{5}$ Thus, in vivo detection of the cancer at an early stage could greatly improve the chances of patient survival by meeting a critical unmet need of accurately differentiating masses from pancreatitis. Optical spectroscopy could be employed via fiber-optic probes guided by imaging such as EUS to provide incremental biochemical and morphological information about tissue to improve diagnostic accuracy.

Reflectance spectroscopy can provide information about tissue morphology (including cell size and density), while endogenous fluorescence can shed light on a tissue's biochemical and morphological framework. These two modes of optical spectroscopy can thus provide complementary information that can be used to study disease in living tissue. Few optical studies have been reported for human pancreatic tissue: optical coherence tomography (OCT) has been employed to identify the neoplastic and non-neoplastic main pancreatic duct layer structures ex vivo, ${ }^{6}$ and a microendoscope has been developed to provide white light reflectance and fluorescence images. ${ }^{7}$ In this report, reflectance and fluorescence spectroscopy were applied to detect and interpret differences between pancreatic tissue types by studying freshly excised human tissues, as well as in vivo mouse xenograft models. To the best of our knowledge, this is the first multimodal approach that incorporates reflectance and fluorescence spectroscopy to distinguish between normal, pancreatitis, and adenocarcinoma tissues using endogenous contrast.

The most common treatment for pancreatic cancer is the Whipple resection, a surgical procedure that involves removing the diseased proximal portion of the pancreas. The procedure can last $7 \mathrm{~h}$ owing to the inaccessibility of the pancreas. Fluorescence and reflectance data were obtained from pancreatic tissues of two patients undergoing Whipple surgery, within 15 min of resection [Fig. 1(a)]. Due to an inherent difficulty in visually differentiating tumor and other tissue types in resected pancreatic tissue, the resected specimen typically contained normal and pancreatitis tissue along with the adenocarcinoma (tumor) tissue. Post-spectroscopy pathology indicated that in patient 1 (2), within a resected sample of $6.5 \times 5 \times 4(6 \times 5.5 \times 3) \mathrm{cm}^{3}$, the tumor was approximately 2 (4) $\mathrm{cm}$ in diameter. On each resected specimen, optical measurements were obtained from five sites. Measurements were repeated to test reproducibility, after which tissue $(0.5 \mathrm{~cm}$ thick) was removed from each site and sent for histological analysis. Pathology indicated that three sites measured on the first patient were pancreatitis, while the other two were normal tissue sites. All five sites measured on the second patient

1083-3668/2007/12(6)/060501/3/\$25.00 @ 2007 SPIE 


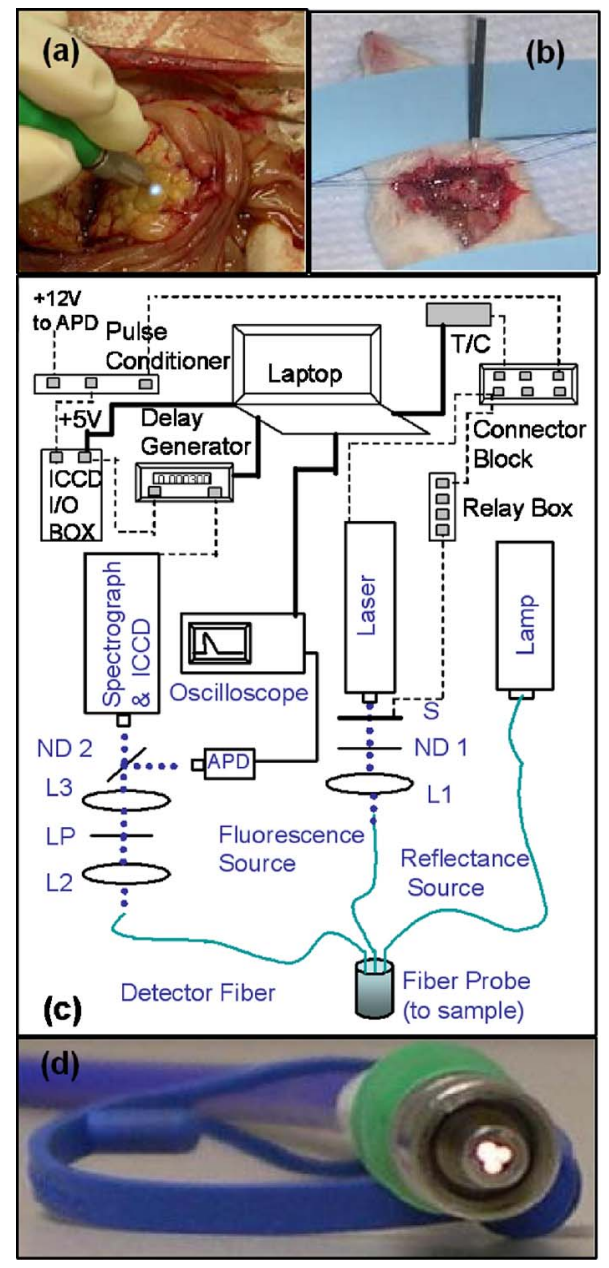

Fig. 1 (a) Human pancreatic tissue during ex vivo measurement. (b) Nude mouse pancreas with human pancreatic cancer xenograft during in vivo measurement. (c) Schematic of the reflectance and fluorescence lifetime spectrometer (RFLS): ND-neutral density filter; $\mathrm{L}$-lens; LP_long pass filter; APD_avalanche photo diode; ICCD_intensified charge coupled device; T/C - timer/counter; $\mathrm{S}$-shutter. Blue dotted lines show optical path, blue solid lines show optical fibers, black dashed lines show electrical connections, and bold black lines show connections to the computer interface. (d) Fiber-optic probe configuration used in human studies. (Color online only.)

were adenocarcinoma tissue sites. The study was approved by the Institutional Review Board, and written consent was obtained from the patients.

Optical measurements were also made on human pancreatic cancer xenografts in nude mice. Experiments on mouse models provided a controlled access and reproducibility not possible in human clinical studies and helped in interpreting the data from human pancreatic tissues. Human pancreatic cancer cells were cultured and injected into the pancreas of two nonobese diabetic/severe combined immunodeficiency mice. After 3 weeks, in vivo fluorescence and reflectance spectra were measured from the grown tumors, within minutes of anesthetizing the mice [Fig. 1(b)]. The animal study was approved by the University of Michigan Committee on Use and Care of Animals.

Data was obtained using a reflectance and fluorescence lifetime spectrometer (RFLS), which was described previously ${ }^{8}$ and recently modified [Fig. 1(c)]. Briefly, for fluo- rescence measurements, a pulsed 355-nm laser (PNV001525140, JDS Uniphase, San Jose, California; $10^{3} \mathrm{~Hz}$, $16 \mu \mathrm{J} /$ pulse, 500-ps pulse duration) was employed for excitation. For reflectance, a tungsten halogen lamp (HL 2000FHSA, Ocean Optics, Dunedin, FL), 360- to 2000-nm emission, was used. Fluorescence and reflectance measurements were made sequentially by blocking light from the other source using shutters. Light from each source was delivered to the sample using separate optical fibers $(600-\mu \mathrm{m}$ core), and the signal from the sample was collected and delivered to detectors via a third identical fiber. At the distal end, the three fibers were placed in a triangular geometry [Fig. 1(d)]. Reflectance and steady-state fluorescence spectra were collected using a spectrograph (MS 125, Oriel Instruments, Stratford, Connecticut) and an intensified charge-coupled device (ICCD) camera (ICCD 2063, Andor Technology, Belfast, Northern Ireland). Time-resolved fluorescence was collected by directing a portion of the emitted fluorescence onto an avalanche photodiode (APD); Hamamatsu, C5658, Bridgewater, New Jersey. All measured spectral data were backgroundsubtracted and corrected for the spectral instrument response. Corrected reflectance data were then scaled by the measured intensity spectrum of the lamp $\left(R_{o}\right)$ to obtain reflectance spectra $\left(R / R_{o}\right)$. All spectra were then normalized by setting the peak intensity value to unity. For each tissue type, mean spectra were calculated from these normalized data.

The plot in Fig. 2(a) shows the average of all normalized fluorescence spectra measured from human pancreatic normal (blue line, $n=4$ ), pancreatitis (orange dotted-dashed line, $n$ $=6$ ), and adenocarcinoma (red dashed line, $n=9$ ) tissues (color online only). Tissue spectra revealed cellular NAD $(\mathrm{P}) \mathrm{H}$ (emission around $460 \mathrm{~nm}$ ) and extracellular matrix collagen (emission peak around $400 \mathrm{~nm})^{8,9}$ The relative collagen emission from adenocarcinoma and pancreatitis tissues was larger than that from normal tissues, as anticipated from the increase in fibrosis in the diseased tissues. ${ }^{4}$ The relative excess of collagen emission from pancreatitis versus normal tissue corresponded well with the increase in fluorescence decay time measured for all pancreatitis tissues versus all normal tissues, ${ }^{3}$ which was attributed ${ }^{10}$ to the longer excited-state lifetime associated with collagen compared to $\mathrm{NAD}(\mathrm{P}) \mathrm{H}$. The gray dotted line shows in vivo fluorescence measured from a human pancreatic cancer xenograft grown in one of the mice. The measured fluorescence peaked around $460 \mathrm{~nm}$, as was expected, since the mice were immunodefficient and so the expected fibrosis usually associated with tumor tissue was absent. Thus, the measured xenograft spectrum could be associated purely with the cellular component of the grown tumor $[$ mainly $\mathrm{NAD}(\mathrm{P}) \mathrm{H}]$ and corresponded well with the fluorescence attributed to the cellular signal in the human study.

The plot in Fig. 2(b) shows the average of all normalized reflectance spectra from human pancreatic normal (blue line), pancreatitis (orange dotted-dashed to line), and adenocarcinoma (red dashed line) tissues. Features in the 400 to 440 and 540 to $580 \mathrm{~nm}$ ranges were attributed to hemoglobin absorption. ${ }^{11}$ The adenocarcinoma sites showed a higher reflectance than pancreatitis and normal tissue sites in the 430 to $500 \mathrm{~nm}$ range. Such tissue reflectance features have been associated with both the density and size of cellular scatterers (e.g., nuclei and organelles). ${ }^{11}$ Indeed, this reflectance feature was observed in the data obtained from xenograft tumor in 

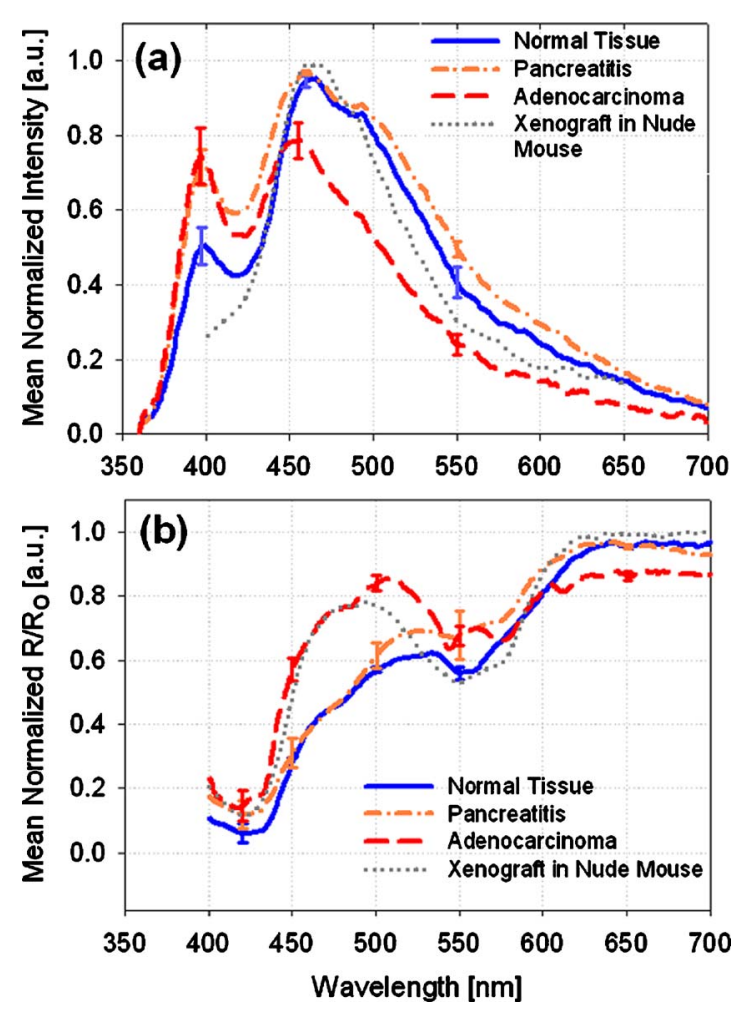

Fig. 2 (a) Mean fluorescence spectra obtained from human pancreatic normal (blue line), pancreatitis (orange dotted-dashed line), and adenocarcinoma (red dashed line) tissues. The fluorescence spectrum of in vivo adenocarcinoma xenograft in a mouse model is also shown (gray dotted line). (b) Mean reflectance spectra obtained from normal (blue line), pancreatitis (orange dotted-dashed line), and adenocarcinoma (red dashed line) tissues. Reflectance spectrum of adenocarcinoma xenograft is also shown (gray dotted line). The standard error is shown at selected wavelengths. (Color online only.)

mice (gray dotted line) and corresponded well with measurements made on human adenocarcinoma.

Figure 3 quantifies some of the major differences observed among spectra by plotting the mean wavelength integrated intensity (area under the curve) of the normalized fluorescence spectra versus the mean ratio of reflectance at $470 \mathrm{~nm}$ to that at $650 \mathrm{~nm}\left(R_{470} / R_{650}\right)$ for each tissue type (normalblue squares; pancreatitis - orange circles; adenocarcinomared triangles; color online only). The plot illustrates one approach suggested by this initial study to quantitatively distinguish among these tissue types via optical spectroscopy.

In conclusion, this limited pilot study suggested that multimodal optical spectroscopy has potential as a method to differentiate between diseased and normal pancreatic tissues. In particular, the results suggest the use of optical diagnostics to distinguish between pancreatic cancer (adenocarcinoma) and non-neoplastic inflammation (pancreatitis), a critical and unmet clinical need. This could also prove useful for real-time tumor margin detection and guidance during pancreatic cancer surgery. Undertaking such a study with a larger patient pool would help further statistically validate these findings. In addition, eventual application of optical spectroscopy via a needle-based probe could help in minimally invasive screening of disease in its early stages, thus resulting in improved

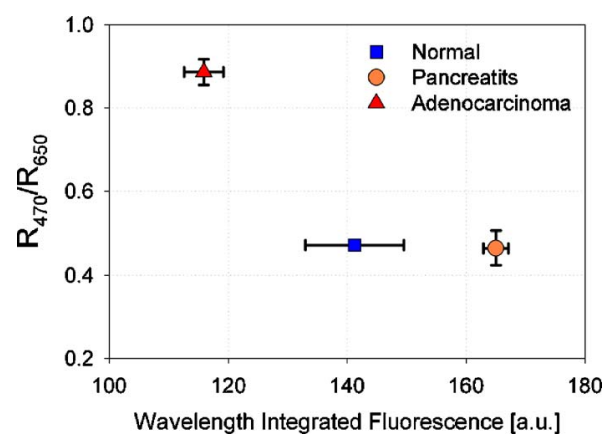

Fig. 3 Plots of mean wavelength integrated fluorescence intensity versus mean ratio of reflectance at $470 \mathrm{~nm}$ to that at $650 \mathrm{~nm}\left(\mathrm{R}_{470} / \mathrm{R}_{650}\right)$ can be used to distinguish between human pancreatic tissue types (normal-blue squares; pancreatitis-orange circles; and adenocarcinoma—red triangles). The error bars indicate the standard error. (Color online only.)

survival rates in the management of pancreatic cancer.

\section{Acknowledgments}

This work was supported by a grant from the University of Michigan Medical School Translational Research Program (to M.-A. M. and J. S.).

\section{References}

1. T. P. Yeo, R. H. Hruban, S. D. Leach, R. E. Wilentz, T. A. Sohn, S. E. Kern, C. A. Iacobuzio-Donahue, A. Maitra, M. Goggins, M. I. Canto, R. A. Abrams, D. Laheru, E. M. Jaffee, M. Hidalgo, and C. J. Yeo, "Pancreatic cancer," Curr. Probl Cancer 26, 176-275 (2002).

2. D. S. Michaud, "Epidemiology of pancreatic cancer," Minerva Chir. 59, 99-111 (2004).

3. M. Chandra, D. Heidt, D. Simeone, B. McKenna, J. Scheiman, and M.-A. Mycek, "Pancreatic tissue assessment using fluorescence and reflectance spectroscopy," in Diagnostic Optical Spectroscopy in Biomedicine IV, D. Schweitzer and M. Fitzmaurice, Eds., Proc. SPIE 6628, 66281R (2007).

4. T. Crnogorac-Jurcevic, E. Efthimiou, P. Capelli, E. Blaveri, A. Baron, B. Terris, M. Jones, K. Tyson, C. Bassi, A. Scarpa, and N. R. Lemione, "Gene expression profiles of pancreatic cancer and stromal desmoplasia," Oncogene 20, 7437-7446 (2001).

5. S. C. Abraham, R. E. Wilentz, C. J. Yeo, T. A. Sohn, J. L. Cameron, J. K. Boitnott, and R. H. Hruban, "Pancreaticoduodenectomy (Whipple resections) in patients without malignancy: are they all 'chronic pancreatitis'?," Am. J. Surg. Pathol. 27, 110-120 (2003).

6. P. A. Testoni, B. Mangiavillano, L. Albarello, P. G. Arcidiacono, A. Mariani, E. Masci, and C. Doglioni, "Optical coherence tomography to detect epithelial lesions of the main pancreatic duct: an ex vivo study," Am. J. Gastroenterol. 100, 2777-2783 (2005).

7. A. L. Kano, A. R. Rouse, S. M. Kroto, and A. F. Gmitro, "Microendoscopes for imaging of the pancreas," in Advanced Biomedical and Clinical Diagnostic Systems II., G. E. Cohn, W. S. Grundfest, D. A. Benaron, and T. Vo-Dinh, Eds., Proc. SPIE 5318, 50-58 (2004).

8. M. Chandra, K. Vishwanath, G. D. Fichter, E. Liao, S. J. Hollister, and M.-A. Mycek, "Quantitative molecular sensing in biological tissues: an approach to non-invasive optical characterization," Opt. Express 14, 6157-6171 (2006).

9. R. Drezek, K. Sokolov, U. Utzinger, I. Boiko, A. Malpica, M. Follen, and R. Richards-Kortum, "Understanding contributions of NADH and collagen to cervical tissue fluorescence spectra: modeling, measurements, and implications," J. Biomed. Opt. 6, 385-396 (2001).

10. K. Vishwanath and M.-A. Mycek, "Do fluorescence decays remitted from tissues accurately reflect intrinsic fluorophore lifetimes?," Opt. Lett. 29, 1512-1514 (2004).

11. I. J. Bigio, S. G. Brown, G. Briggs, C. Kelley, S. Lakhani, D. Picard, P. M. Ripley, I. G. Rose, and C. Saunders, "Diagnosis of breast cancer using elastic-scattering spectroscopy: preliminary clinical results," J. Biomed. Opt. 5, 221-228 (2000). 